\title{
Masses and Beta-decay Studies of Neutron-rich Nuclei using the X-array and Gammasphere
}

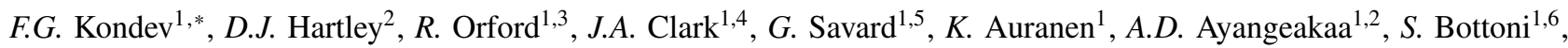

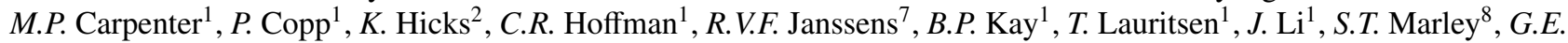

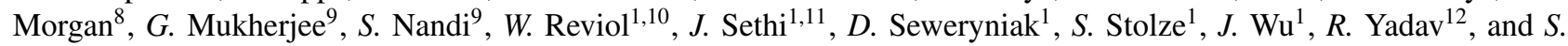 \\ $\mathrm{Zhu}^{1}$
}

${ }^{1}$ Physics Division, Argonne National Laboratory, Lemont, Illinois 60439, USA

${ }^{2}$ Department of Physics, U.S. Naval Academy, Annapolis, Maryland 21402, USA

${ }^{3}$ Nuclear Science Division, Lawrence Berkeley National Laboratory, Berkeley, California 94720, USA

${ }^{4}$ Department of Physics and Astronomy, University of Manitoba, Winnipeg, Manitoba R3T 2N2, Canada

${ }^{5}$ Department of Physics, University of Chicago, Chicago, Illinois 60637, USA

${ }^{6}$ Universitet degli Studi di Milano and INFN, Via Celoria 16, 20133 Milano, Italy

${ }^{7}$ Department of Physics and Astronomy, University of North Carolina, Chapel Hill, North Carolina 27599, USA and Triangle Universities Nuclear Laboratory, Duke University, Durham, North Carolina 27708, USA

${ }^{8}$ Department of Physics and Astronomy, Louisiana State University, Baton Rouge, Louisiana 70803, USA

${ }^{9}$ Variable Energy Cyclotron Centre, 1/AF Bidhan Nagar, Kolkata 700064, India and Homi Bhabha National Institute, Training School Complex, Anushaktinagar, Mumbai-400094, India

${ }^{10}$ Department of Chemistry, Washington University, St. Louis, Missouri 63130, USA

${ }^{11}$ Department of Chemistry and Biochemistry, University of Maryland, College Park, Maryland 20742, USA

${ }^{12}$ Department of Biological and Physical Sciences, South Carolina State University, Orangeburg, South Carolina 29117, USA

\begin{abstract}
.
Properties of neutron-rich nuclei in the A 160 region are important for achieving a better understanding of the nuclear structure in this region where little is known owing to difficulties in the production of these nuclei at the present nuclear physics facilities. These properties are essential ingredients in the interpretation of the rareearth peak at $\mathrm{A} \sim 160$ in the $r$ process abundance distribution, since theoretical models are sensitive to nuclear structure input. Predicated on these ideas, we have initiated a new experimental program at Argonne National Laboratory. During the first experiment, beams from the Californium Rare Isotope Breeder Upgrade radioactive beam facility were used in conjunction with the SATURN decay station and the X-array. We focused initially on several odd-odd nuclei, where $\beta$ decays of both the ground state and an excited isomer were investigated. Because of the spin difference, a variety of structures in the daughter nuclei were selectively populated and characterized based on their decay properties. Mass measurements using the Canadian Penning Trap aimed at establishing the excitation energy of the $\beta$-decaying isomers were also carried out. Evidence was found for a change in the single-particle structure, which in turn results in the formation of a sizable $\mathrm{N}=98$ sub-shell gap at large deformation. Results from the first experimental campaign using the newly-commissioned $\beta$-decay station at Gammasphere are also presented.
\end{abstract}

\section{Introduction}

The structure of deformed, neutron-rich nuclei in the rareearth region is of significant interest for both the nuclearstructure and astrophysics fields. Although much progress is being made in our understanding of the $r$ process, a satisfactory explanation for the elemental peak in abundance near $A=160$ is still elusive [1]. The quest to understand the origin of this peak may be key to correctly identifying the astrophysical conditions for the $r$ process. Theoretical models of element production are dependent on masses and lifetimes of neutron-rich, deformed rare-earth nuclei in this region where little or no information is avail-

*e-mail: kondev@anl.gov able. The available nuclear-structure information is also scarce, owing to difficulties in the production of these nuclei. In order to address these issues, an experimental program has been initiated at Argonne National Laboratory using high-purity radioactive beams produced by the CAlifornium Rare Isotope Breeder Upgrade (CARIBU) facility. Mass measurements using the Canadian Penning Trap (CPT) and $\beta-\gamma-\gamma$ coincidence studies using the Scintillator And Tape Using Radioactive Nuclei (SATURN) moving tape system and the $\mathrm{X}$-array spectrometer were carried out. During the first experimental campaign, several longlived, $\beta$-decaying states were identified in the neutron-rich ${ }^{160} \mathrm{Eu}$ and ${ }^{162} \mathrm{Eu}$ nuclei [2]. Evidence was found for a change in the single-particle structure, which in turn re- 


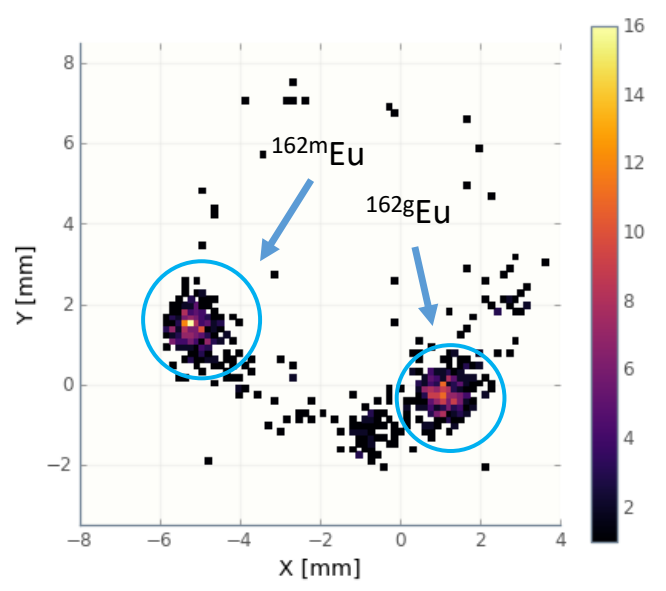

Figure 1. A histogram of a phase-imaging measurement showing the positions of ${ }^{162 m, g} \mathrm{Eu}$ ions on the detector plane.

sults in the formation of a sizable $\mathrm{N}=98$ sub-shell gap at large deformation [2].

The nuclear applications community is also interested in the decay properties of nuclei in this fissionproduct region. Past studies using the high-resolution $\gamma$ ray spectroscopy technique usually involved a single, or a few, germanium detectors, which resulted in incomplete decay schemes. Recently, measurements using the calorimetric Total Absorption Gamma-ray Spectroscopy (TAGS) method (see for example Refs. [3-5] and references therein) provide useful information on average $\beta$ decay properties. However, the technique utilizes lowresolution $\mathrm{NaI}$ detectors and depends sensitively on detailed knowledge of the decay schemes, which are usually not well known. As a consequence, TAGS relies on extensive theoretical considerations and Monte-Carlo simulations, which contribute to results that are model dependent and uncertainties are not well determined. We report here on the first $\beta-\gamma-\gamma$ spectroscopy measurements using the newly-commissioned $\beta$-decay station at Gammasphere, where both the discrete and calorimetric approaches can simultaneously be used.

\section{Mass measurements with the CPT and decay spectroscopy studies using the X-array}

The mass and $\beta$-decay spectroscopy experiments were both conducted in the low-energy experimental area of the CARIBU facility [6] located at the ATLAS facility at Argonne National Laboratory. The CARIBU beams were further purified by a Multi-Reflection Time-Of-Flight (MR-TOF) mass separator [7] before being delivered to the CPT mass spectrometer [8], where the Phase-Imaging Ion-Cyclotron-Resonance (PI-ICR) technique [9, 10] was used. From the new results, isomers in both ${ }^{160} \mathrm{Eu}$ and ${ }^{162} \mathrm{Eu}$ were established for the first time at excitation energies of 93.0 (12) $\mathrm{keV}$ and 160.2 (24) keV, respectively. It is worth noting that previous studies of ${ }^{160} \mathrm{Eu}$ and ${ }^{162} \mathrm{Eu}$

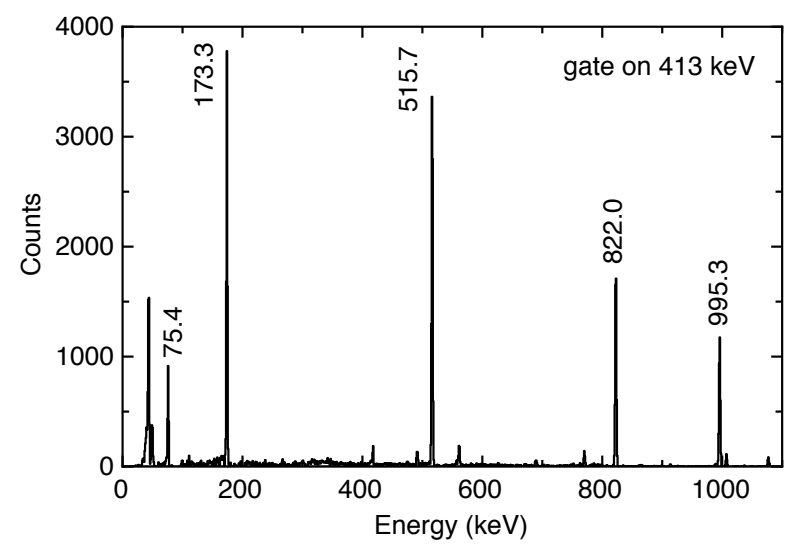

Figure 2. Gamma rays in coincidence with the 413-keV $\gamma$ ray following the decay of the high-spin, $\beta$-decaying state in ${ }^{160} \mathrm{Eu}$.

postulated the existence of a single $\beta$-decaying state [1113]. An example of the final phase measurement showing the well-resolved ${ }^{162 m, g} \mathrm{Eu}^{2+}$ ions is given in Figure 1.

The nuclear-structure properties of the ground and isomeric states in ${ }^{160} \mathrm{Eu}$ and ${ }^{162} \mathrm{Eu}$ were subsequently investigated through $\beta$-decay measurements. The CARIBU beams were directed onto a $\beta$-decay counting station comprised of the SATURN moving-tape system, four scintillator detectors for $\beta$-particle detection, and the X-array spectrometer with four germanium clover detectors, and one Low Energy Photon Spectrometer (LEPS), for $\gamma$-ray detection [14].

Combining the newly obtained experimental data with predictions of multi-quasiparticle calculations, the structure of the ground- and the isomeric states in ${ }^{160} \mathrm{Eu}$ and ${ }^{162} \mathrm{Eu}$ were assigned the $\mathrm{K}^{\pi}=5^{-}$(g.s.) and $0^{-}$(isomer) states of the $\pi 5 / 2[413] \otimes v 5 / 2[523]$ configuration and the $\mathrm{K}^{\pi}=1^{+}$(g.s.) and $6^{+}$(isomer) states of the $\pi 5 / 2[413] \otimes v 7 / 2$ [633] configuration, respectively [2]. In ${ }^{160} \mathrm{Eu}$, the $\mathrm{K}^{\pi}=5^{-}$ground state was found $[2,15]$ to preferentially $\beta$ decay via an allowed transition to the $\mathrm{K}^{\pi}=5^{-}, \pi^{2}(5 / 2[413], 5 / 2[532])$ state at $1999 \mathrm{keV}$ in the daughter nucleus ${ }^{160} \mathrm{Gd}$. Such a transition involves the $v 5 / 2[523] \rightarrow \pi 5 / 2[532]$ configuration change and it is very fast. The $\mathrm{K}^{\pi}=5^{-}$state in ${ }^{160} \mathrm{Gd}$ has multiple $\gamma$-ray decay branches, the strongest one being via the $516-\mathrm{keV}$ transition to the newly observed $\mathrm{K}^{\pi}=4^{+}$, $\pi^{2}(5 / 2[413], 3 / 2[411])$ state at $1483 \mathrm{keV}[2,15]$. The latter subsequently decays via the $413-\mathrm{keV}$ transition to the previously known $\mathrm{K}^{\pi}=4^{+}$state at $1071 \mathrm{keV}$ which has a dominant $v^{2}(3 / 2[521], 5 / 2[523])$ configuration [15]. A sample $\gamma$-ray spectrum produced by gating on the $413-\mathrm{keV}$ transition is shown in Figure 2.

In ${ }^{162} \mathrm{Eu}$, only $\beta$ decay of the high-spin isomer was observed. It predominately feeds the newly observed $\mathrm{K}^{\pi}=\left(6^{-}\right), v^{2}(5 / 2[523], 7 / 2[633])$ state in ${ }^{162} \mathrm{Gd}$ at $1453 \mathrm{keV}$ that depopulates via the 206- and $331-\mathrm{keV} \gamma$ rays to the $\mathrm{I}^{\pi}=\left(6^{+}\right)$and $\left(5^{+}\right)$members of the $\mathrm{K}^{\pi}=2^{+} \gamma$-vibrational band, respectively [2]. 


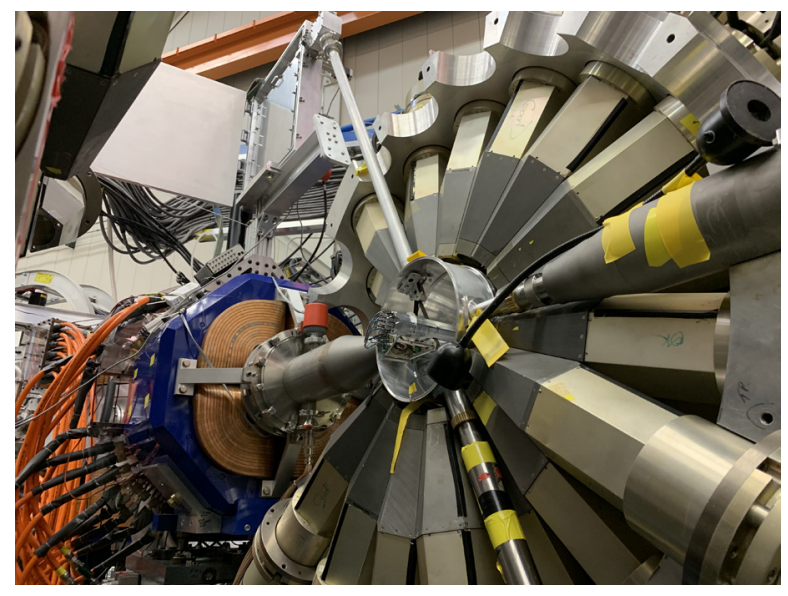

Figure 3. The new $\beta$-decay station at Gammsphere showing the tape-transport system and the array of six plastic scintillator detectors for $\beta$-particle triggering.

\section{Decay spectroscopy studies using Gammasphere}

Gammasphere is one of most powerful $\gamma$-ray spectrometers in the world, owing to its good energy resolution, high efficiency and exceptional resolving power. The calorimetric potential of Gammasphere has only been used in the past for measuring entry regions in heavy nuclei [16] and never for $\beta$-decay studies. In principle, Gammasphere should be far superior compared to any TAGS device, as it retains all the information from many decay paths, instead of adding them up inside the detector. These data can be combined in many ways in the off-line analysis, including the performance of calorimetric studies for the highenergy part of the decay energy spectrum simultaneously with the discrete $\gamma$-ray spectroscopy data. In addition, the high coincidence power of Gamasphere allows dedicated $\gamma-\gamma$ angular correlations to be studied, which can provide information on the $\gamma$-ray transition multipolarities. Such data allow spins and parities of the excited states to be determined and this information is needed in the analysis of both the discrete and calorimetric $\beta$-decay data.

One reason that Gammasphere has never been used in $\beta$-decay spectroscopy studies in the past is the so-called "parent-daughter" problem: any interesting and exotic nucleus produced far from stability in a reaction will quickly decay to a series of much longer-lived daughter and granddaughter isobars that rapidly build up to secular equilibrium with much higher activity. As a consequence, the long-lived activities quickly swamp the exotic decay of interest. A solution to this problem is a tape-transport system which can be used to implant the CARIBU beams into the center of the spectrometer for an optimal collection time (usually 1-5 half-lives of the nuclide of interest) and then to remove the accumulated activity after a suitable counting time (usually 5-10 half-lives).

A new $\beta$-decay counting station for optimal use with Gammasphere was recently completed and commissioned. It comprises of a tape-transport system and an array of 6 plastic scintillators for $\beta$-particle triggering [17], as shown
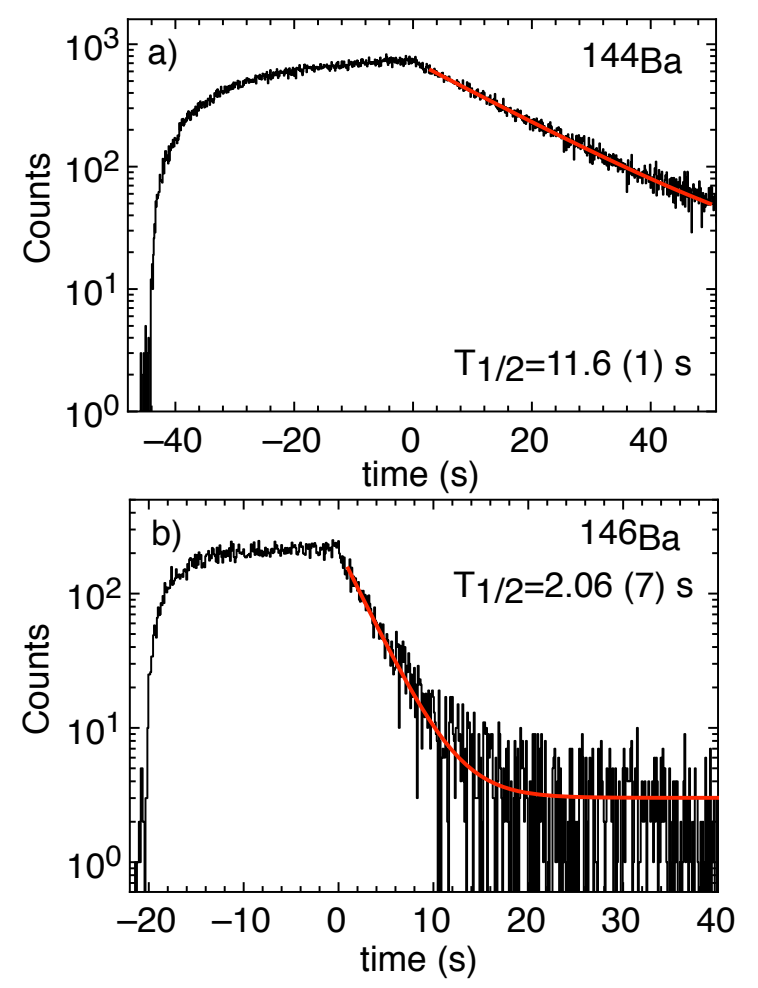

Figure 4. Time spectra produced by gating on: (a) - sum of 104-, 157-, 173- and 388-keV $\gamma$ rays following the ${ }^{144} \mathrm{Ba}$ decay; (b) sum of 121-, 141-, 197-, 232- and 251-keV $\gamma$ rays following the ${ }^{146}$ Ba decay.

in Figure 3. During the commissioning experiment, data was collected using CARIBU beams of several $A=144$ $\left({ }^{144} \mathrm{Ba}\right.$ and $\left.{ }^{144} \mathrm{La}\right)$ and $\mathrm{A}=146\left({ }^{146} \mathrm{Ba},{ }^{146 m, g} \mathrm{La}\right.$ and $\left.{ }^{146} \mathrm{Ce}\right)$ nuclei. Figure 4 shows time spectra produced by gating on selected $\gamma$-ray transitions following $\beta$ decays of ${ }^{144} \mathrm{Ba}$ and ${ }^{146} \mathrm{Ba}$. The measured half-lives of $11.6(1) \mathrm{s}\left({ }^{144} \mathrm{Ba}\right)$ and $2.06(7) \mathrm{s}\left({ }^{146} \mathrm{Ba}\right)$ are in good agreement with the presently evaluated values $[18,19]$.

The neutron-rich ${ }^{146} \mathrm{La}$ nuclide is of interest to several decay-data applications, including decay heat from nuclear reactors and antineutrino spectra reconstructions [20]. This nucleus is known to have $\beta$-decaying ground and isomeric states that were assigned $\mathrm{I}^{\pi}=\left(2^{-}\right)$ and $\left(6^{-}\right)$, respectively, based on a shell-model predictions [19]. However, the excitation energy of the isomer was not known and the decays of the two states were not well separated, thus resulting in incomplete and misleading level schemes. Controversially, while the decay data for the high-spin, $\beta$-decaying state in ${ }^{146} \mathrm{La}$ are included in the ENSDF database [19], they are absent in the NUDAT (claimed to be a "friendly face" of ENSDF) and ENDF/BVIII.0 [21], and JEFF3.3 [22] libraries, which are commonly used by the nuclear applications community. Using the newly-collected data, we were able to separate and significantly improve the decay schemes for the $\beta$ decaying ground and isomeric states in ${ }^{146} \mathrm{La}$. A $\gamma$-ray spectrum showing transitions in coincidence with the 503$\mathrm{keV} \gamma$ ray, the $6^{+}$to $4^{+}$transition within the ground-state band of ${ }^{146} \mathrm{Ce}$, is shown in Figure 5. The excitation en- 


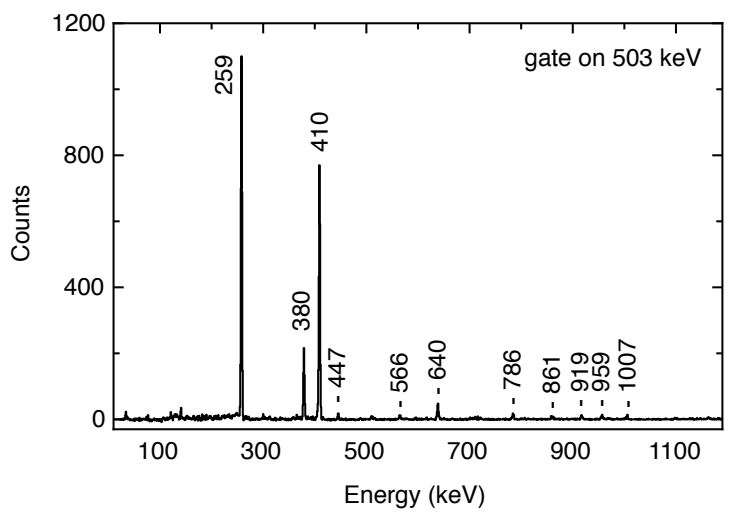

Figure 5. Gamma-ray spectrum from the high-spin $\beta$-decay of ${ }^{146} \mathrm{La}$ showing the main transitions in prompt coincidence with the 503-keV $\gamma$ ray, depopulating the $\mathrm{I}^{\pi}=6^{+}$level of the groundstate band in ${ }^{146} \mathrm{Ce}$ daughter nucleus.

ergy of the isomer was recently established as 141.4(25) $\mathrm{keV}$ using direct mass measurements at the CPT and the phase-imaging technique [10].

The decay scheme of ${ }^{144}$ La was also significantly improved and comparisons with the previous TAGS data [3] shows some notable differences, although it is worth pointing out that no uncertainties were given in Ref. [3].

This work is funded by the U.S. Department of Energy, Office of Nuclear Physics, under Contract No. DE-AC0206CH11357 (ANL) and by the National Science Foundation under Grant Nos. PHY-1502092 (USNA). The work was also sponsored in part by the U.S. Department of Energy, National Nuclear Security Administration, Office of Defense Nuclear Nonproliferation R\&D (NA-22). This research used resources of Argonne National Laboratory's ATLAS facility, which is a DOE Office of Science User Facility.

\section{References}

[1] M.R. Mumpower, G.C. McLaughlin, R. Surman, and A.W. Steiner, J. Phys. G: Nucl. Part. Phys. 44, 034003
(2017).

[2] D.J. Hartley et al., Phys. Rev. Lett. 120, 182502 (2018).

[3] R.C. Greenwood et al., Nucl. Instr. and Meth. Phys. Res. A390, 95 (1997).

[4] B.C. Rasco et al., Phys. Rev. C95, 054328 (2017).

[5] E. Valencia et al., Phys. Rev. C95, 024320 (2017).

[6] G. Savard, S. Baker, C. Davids, A. Levand, E. Moore, R. Pardo, R. Vondrasek, B. Zabransky, and G. Zinkann, Nucl. Instr. and Meth. Phys. Res. B266, 4086 (2008).

[7] T. Hirsh et al., Nucl. Instr. and Meth. Phys. Res. B376, 229 (2016).

[8] J. Van Schelt et al., Phys. Rev. Lett. 111, 061102 (2013).

[9] S. Eliseev, K. Blaum, M. Block, C. Droese, M. Goncharov, E. Minaya Ramirez, D. A. Nesterenko, Yu. N. Novikov, and L. Schweikhard, Phys. Rev. Lett. 110, 082501 (2013).

[10] R. Orford et al., Nucl. Instr. and Meth. Phys. Res. B, in press.

[11] C.W. Reich, Nucl. Data Sheets 105, 557 (2005).

[12] C.W. Reich, Nucl. Data Sheets 108, 1807 (2007).

[13] J. Wu et al., Phys. Rev. Lett. 118, 072701 (2017).

[14] A.J. Mitchell et al., Nucl. Instr. and Meth. Phys. Res. A763, 232 (2014).

[15] D.J. Hartley et al., to be published.

[16] P. Reiter et. al., Phys. Rev. Lett. 84, 3542 (2000).

[17] P. Copp et al., to be published

[18] A.A. Sonzogni, Nucl. Data Sheets 93, 599 (2001).

[19] Yu. Khazov, A. Rodionov, and G. Shulyak, Nucl. Data Sheets 136, 163 (2016)

[20] P. Dimitriou and A.L. Nichols, Total Absorption Gamma-ray Spectroscopy for Decay Heat and Other Applications (INDC(NDS)-0676, IAEA, Vienna, February 2015) 1-36.

[21] NUDAT and ENDF databases at www.nndc.bnl.gov.

[22] JEFF3.3 database at www.oecd-nea.org/dbdata. 